\title{
Evaluation of a motivational pre-exercise music intervention
}

Corresponding author: Rachel Hallett, Room 2.058 Grosvenor Wing, Faculty of Health, Social Care and Education, Kingston University and St George's University of London, St George's Hospital, Blackshaw Road, Tooting, London SW17 0QT

Email: r.hallett@sgul.kingston.ac.uk

This research was carried out while the corresponding author was at Keele University.

Second author: Alexandra Lamont, School of Psychology, Keele University, Keele, Staffordshire ST5 5BG, UK. Tel. +44 (0)1782 733323 Fax +44 (0) 1782733387 Email: a.m.lamont@keele.ac.uk

\begin{abstract}
While music has been found to motivate exercisers during workouts, its potential as a pre-exercise motivator has rarely been investigated. This study evaluated a self-selected, pre-exercise music intervention against implementation intentions (writing down 'if... then...' sentences relating to overcoming barriers) and a control condition. Fifty participants $\left(M_{\text {age }}=43\right)$ took part in a longitudinal, randomised, between-participants study, from 99 recruited. For both interventions, participants had significantly more success meeting self-set exercise goals than the control group, and the music group exercised significantly more frequently than the control group. There was support for music as a comparable intervention to implementation intentions.
\end{abstract}

\section{Keywords}

Adherence, affect, exercise behaviour, interventions, strategies 


\section{Introduction}

Motivation to exercise is addressed in numerous health psychology studies. The motivational effect of playing music during exercise is well-recognised, and includes reduced perceived exertion, improved affect, increased output and more efficient energy use (Karageorghis and Priest, 2012), yet music cannot be played during exercise if the exercise does not take place. In this study, we explore music's potential as a preexercise motivator to help convert intention to behaviour.

Exercise lowers the risks of developing mental (Donaghy, 2007; Stanton et al., 2014) and physical (Myers, 2008; Warburton et al., 2010) health issues. However, many people struggle to meet the recommended levels of physical activity, with around $60 \%$ of men and $70 \%$ of women in England participating in sport or active recreation less than once per week (Sport England, 2013).

Health behaviour research indicates a 'gap' between intention and action. The conversion rate of intention into a healthy behaviour is around 50\% (Ajzen and Fishbein, 2005; Sniehotta, 2013), although Sniehotta notes that these findings relate to short studies, and as people attempt to change behaviour over longer periods, the conversion figure is closer to $10-20 \%$ (2013). Research has examined cognitive-based interventions to address this, such as implementation intentions (Gollwitzer, 1999), where participants identify perceived barriers to a behaviour and create 'If... then...' sentences (such as 'If I don't feel like exercising, then I will remind myself how good I'll feel afterwards'), then write these down. This is a self-regulatory strategy, intended to overcome challenges such as distractions or goal conflict. Recognised barriers act as environmental cues and, through association with behaviours to overcome them, reduce the cognitive effort required to deal with them (Gollwitzer and Oettingen, 1998), possibly introducing a level of automaticity (Gollwitzer and Sheeran, 2006). 
Implementation intentions' effectiveness for health behaviours is well-supported (Gollwitzer and Sheeran, 2006). There is some difference in meta-analyses: Gollwitzer and Sheeran's meta-analysis found a medium to large effect in 5 out of 6 physical activity studies, with a very large effect in two, while Bélanger-Gravel et al. (2011) found a more modest effect in their meta-analysis of 26 exercise studies, finding a small to medium effect. Nevertheless, both meta-analyses find support for implementation intentions, and it offers a straightforward intervention that can be self-delivered.

Music may also help motivate exercisers. Biochemical, physiological and physical responses to music are well-documented (Hodges, 2009), and many exercisers and athletes use music to increase arousal levels during physical activities (Hallett \& Lamont, 2015; Laukka and Quick, 2013).

In 1999, Karageorghis et al. proposed a theoretical framework for music's motivational effect in exercise, suggesting that it may assist adherence, although research has focused on performance outcomes during exercise, rather than adherence (Karageorghis and Priest, 2012). Adherence is not well-defined in health behaviour literature. Vrijens et al. (2012) note, in a systematic review of adherence to medications, that terminology and quantification is inconsistent. They identify three main phases: initiation, implementation and discontinuation, and note various statistics that are used relating to the amount and regularity with which medication is taken and periods between doses. These concepts carry over to exercise to some extent, although the 'prescription' may vary considerably between self-set goals, fitness instructors' or health professionals' recommendations, or an ad hoc approach where exercise is fitted around other commitments as they arise, with little planning ahead. Adherence measures of exercise may therefore include absolute frequency, frequency as a percentage of an exercise goal, and 'dose' in terms of time and intensity. 
Adherence increases that may be attributable to music use have been identified mainly in men: Hallett (2015) found that gym exercise frequency correlated with the extent to which they listened to their own music while working out, but found no association for women. Alter et al. (2015) found a link between adherence and listening to music for cardiac rehabilitation patients, but $77 \%$ of their intervention group and $56 \%$ of their control group were male, which may have led to their results reflecting gender differences.

While music played during exercise appears to help boost output for intense and moderate activities (Clark et al., 2015; Elliott et al., 2005; Olson et al., 2015; Stork et al., 2014), and increase adherence (Alter et al., 2015; Hallett, 2015), there are also grounds to speculate that music played before exercise might help bridge the intentionbehaviour gap. Juslin and Sloboda note that "listeners use music to change emotions... most people experience music (somehow, somewhere) every day of their lives, often with an accompanying affective reaction of some sort" (2010, p.3), and the emotional effect of music appears to relate to levels of physiological arousal (Rickard, 2004). Music can affect physiological responses including electrodermal activity, heart rate and respiration rate (Salimpoor et al., 2009), and may also influence hormone and neurotransmitter levels (Hodges, 2009).

Although few studies have looked at music-listening before physical activity, links with arousal have been observed; young tennis players reported increased arousal levels alongside more positive mood when they listened to music before playing a match (Bishop et al., 2007). Research beyond sport and exercise finds music used to increase arousal for activities such as housework which might hold little appeal otherwise (Sloboda et al., 2001), and North et al. (2004) note that "people do indeed consciously and actively use music in different interpersonal and social contexts in 
order to produce different psychological states" (p. 75). This suggests a process of selecting and playing music as a precursor to a desired state. Music use before rather than during exercise may therefore be key to adherence.

A possible mechanism by which pre-exercise music might lead to adherence involves a combination of cognitive processes and an increase in positive affect (Clark et al., 2015), which in turn prevents ego depletion (Tice et al., 2007; Zhu et al., 2015) and its accompanying reduction in self-control, or willpower (Baumeister et al., 2007). Since happy music can increase positive affect (Saarikallio et al., 2013), there would be no requirement for physical activity's affect-improving effect for an improvement in willpower, and hence the initiation stage of Vrijens et al.'s (2012) adherence model. Juslin et al.'s BRECVEMA model (2013) presents eight levels at which music behaves as a stimulus resulting in physiological and cognitive responses. The second level of the model, rhythmic entrainment, involves physiological processes such as heartrate becoming matched to the beat of the music, while the third level, emotional contagion, sees the emotion expressed within the music mirrored by the individual. It appears that 'happy' music creates positive affect, decreasing ego depletion and facilitating willpower (Saarikallio et al., 2013; Tice et al., 2007; Zhu et al., 2015). The mechanism underpinning implementation intentions, described previously, is predominantly cognitive. Both interventions ease the conversion of intention to behaviour, although through somewhat different routes; music appears to act on a broader range of responses than implementation intentions, so may be more effective.

Exercise music research has tended to use musical stimuli selected by researchers or participants' peers, rather than self-selected music. Mitchell and MacDonald (2012) have questioned such approaches, following their own experiences with pain management studies: "personal preference can render varied types of music 
'functionally equivalent.' That is, the participants chose music with contrasting structural features to produce the same effect" (p. 234). This suggests that music selfselected to meet certain criteria, while resulting in diverse stylistic traits across stimuli, may create a more consistent effect than all participants listening to the same music track. DeNora (2000) has found participants are highly adept at choosing music that is appropriate for their situation, while Thoma et al. (2012) identified a propensity for and ability to select music with appropriate emotional congruence for specific situations, further emphasising the case for autonomy in music choice.

It is therefore suggest that self-selected music, played at a point where the individual is struggling with willpower to start a workout, may have an effect on physiology and affect that helps facilitate willpower and overcome barriers, leading to implementation of exercise behaviour. Repetition of the strategy establishes adherence. The point of struggle could also be the trigger for an implementation intentions strategy to be invoked, working cognitively. Comparing these two approaches is worthy of study as they could act at a similar point and offer strategies for the unmotivated exerciser. Given music has a cognitive and physiological effect, while implementation intentions are a cognitive strategy, it might be expected that music has a greater effect. Additionally, music is recognised as pleasurable (North et al., 2004).

Inconsistencies in the adherence literature have been noted, while recognising the importance of concepts including prescription, implementation and dose (Vrijens et al, 2012). Rhodes et al. (2003) compared exercise behaviour to self-set goals, and it is proposed this study does likewise. Based on the adherence literature, dose (time and absolute frequency) should also be considered.

We devised a study to investigate whether self-chosen pre-exercise music could increase exercise adherence, using multiple adherence measures. Our hypotheses were: 
(H1) Both the intervention groups would carry out more exercise than the control group on all measures; and

(H2) Music would have a greater effect than implementation intentions because it is likely to provoke a wider range of physiological and cognitive responses.

\section{Method}

\section{Design}

A randomised, controlled, between-participants design was used, with three conditions, examining the effects of interventions over a 6 month period. The first condition was a music intervention, with participants instructed to choose and play music to 'get them in the mood' to exercise, coinciding with the point at which low motivation was most likely to override their intentions. Self-selected music was used for consistency of effect in accordance with Mitchell and MacDonald (2012). The second condition used implementation intentions, with participants identifying potential barriers to exercise and devising 'if... then...' sentences to overcome these, which they were asked to write down prior to the study. An example might be "If I don't feel like going to the gym, then I will remind myself how good I feel when I've finished my workout." Each survey also asked the second group what sentences they had used. The third condition was a control condition with no intervention. The control group was asked about their exercise plans at the outset and reported their exercise using online surveys, as with the other groups, but did not have any pre-exercise tasks. Participants chose their exercise activities and the frequency with which these were carried out, in order to accommodate a range of fitness levels and lifestyles. 


\section{Participants}

There were 99 participants (77 women, 22 men), mean age 40.9 years $(S D=13.3)$.

Thirty had not exercised in the previous six months, while 69 were regular exercisers. Fifty participants completed the study, with most attrition in the first month. Of the 50, 38 were regular exercisers at the outset, with 12 not exercising regularly. Attrition was therefore $60 \%$ for those not exercising regularly prior to the outset, and $45 \%$ for regular exercisers. Initial power for 99 participants was calculated at .94 for a large effect at $\alpha=$ .05 , but fell to .71 with the attrition.

\section{Apparatus}

Data were collected using online surveys through SmartSurvey. Sign-up surveys were used to collect informed consent and background information. Report surveys were completed every 4 weeks for exercise activities across one week. Participants provided an email address in the sign-up survey, after which all information was provided via email.

\section{Materials and measures}

Four different measures of adherence were analysed: Goal achievement (actual sessions as a percentage of planned sessions); Duration; Days exercised on; and Sessions, the number of workouts per week. The results were also compared with current UK exercise guidelines.

Participants signed up to take part in the study by completing an online survey, detailing activities and the frequency (times per week) with which they planned to carry each one out. Individual exercise targets measured in sessions per week were calculated 
for comparison with actual activities. Participants chose their own exercise activities to ensure ecological validity and appropriateness, and to allow autonomous goals and outcomes to be compared with official exercise recommendations. Data was also collected regarding participants' exercise in the period prior to the survey, by asking how long they had been exercising regularly, or, for non-exercisers, how long since they had exercised regularly, with the option to state they had never undertaken regular exercise. Those who had not exercised regularly in the previous 6 months were considered to be non-exercisers. Personality was measured using the Ten Item Personality Inventory (Gosling et al., 2003), and participants provided data for their age in years and their gender.

Once every four weeks, over a period of 6 months ${ }^{1}$, participants completed surveys, detailing their exercise sessions. For each survey, Duration (minutes per week), Days (days per week on which exercise took place), Sessions (total number of workouts per week) and Goal Achievement (Sessions expressed as a percentage of targets set in the participant's initial survey) were calculated. Exercise sessions lasting fewer than 10 minutes were excluded as this is the minimum duration of an exercise session stipulated in current guidelines (NHS, 2011). Non-strenuous physical activities which did not meet the criteria set out by the ACSM (American College of Sports Medicine: Garber et al., 2011) were excluded from one survey (painting, which had not been included in the

\footnotetext{
${ }^{1}$ A small number of participants, who were returning to exercise after a break rather than already exercising, continued completing surveys for 12 months in order to accommodate the period in which discontinuation was most likely to occur (Sperandei et al., 2016). For this report, analysis was carried out for 6 months of data, as regularly exercising participants only took part for that period.
} 
initial plan) while gardening was included as it is recognised as being sufficiently strenuous and was included in several exercise plans. For data analysis, the four adherence measures - Duration, Days, Sessions and Goal Achievement - were averaged across all completed surveys for each participant.

Data was also collected on music used, which was categorised according to Rentfrow and Gosling's four categories (2003): Reflective and Complex, Intense and Rebellious, Upbeat and Conventional, and Energetic and Rhythmic. Participants provided their implementations intentions sentences, which were analysed as 'stick' or 'carrot' using terminology from Stone et al. (2009), reflecting positive and negative incentivisation respectively. Participants provided data on their height and weight at baseline, and their weight at the three month and six month points for calculation of BMI.

\section{Procedures}

Participants were recruited by handing out flyers at gyms and a parkrun (www.parkrun.org.uk), through social media (Twitter and Facebook), coverage in the local press, and publicising the study on the Fetch (www.fetcheveryone.com) and Myfitnesspal (ww.myfitnesspal.com) websites. All recruitment materials provided links to further information and a sign-up survey.

On receipt of a completed survey, the participant was allocated to a condition: these were assigned in the order in which completed surveys were received (music, implementation intentions, control, music, and so on). Participants were allocated to a group the Sunday after signing up and received a message with instructions according to their condition. Those allocated to the music intervention condition were asked to play 
music to 'get them in the mood' prior to exercise, coinciding with the point at which lack of motivation was most likely to override their intentions. Those allocated to the implementation intentions condition were asked to think of reasons that they might not start a planned exercise session, then to consider ways to overcome these barriers, and asked to write down for their own reference 'if..., then...' sentences for each one. All participants were advised that they would shortly be invited to log and report their exercise.

On the Thursday following allocation, participants were asked to prepare to record their exercise over the following week. Four days later, on the Monday, they received a reminder to make a note of their activities, and the following Monday, a reminder to complete the online survey. Further reminders were sent each Monday if surveys had not been completed. If two consecutive surveys were uncompleted, the participant was assumed to have withdrawn and not contacted further. Withdrawal did not necessarily constitute discontinuing exercise: blogs on websites from which participants had been recruited indicated that some participants who had withdrawn were continuing to exercise. Data was therefore analysed only for those completing the study.

Due to the data being collected over a period of 6 months, participants sometimes had to complete the next available survey due to holidays and illness. Where chronic conditions occurred, participants withdrew, and some participants discontinued participation due to, for example, work and family commitments. The majority of those who did not complete the study did not provide reasons but discontinued responding to emails requesting completion of surveys. 


\section{Analysis}

Duration, Days and Sessions were analysed using one-way ANOVAs to compare the three groups. Where Levene's test indicated that the assumption of homogeneity of variance was violated, the Welch statistic was used (Pallant, 2010; Tomarken and Serlin, 1986). The Goal Achievement data was proportional, and as some participants carried out more than their intended exercise, some Goal Achievement scores were over $100 \%$. Because of this, arcsine transformations, the usual approach for proportional data (Cohen and Cohen, 1983), were not possible. Analysis of Goal Achievement was therefore carried out using a Kruskal-Wallis test to avoid the effect of weighting, and medians rather than mean scores are reported.

\section{Ethics}

Ethical approval was granted by Keele University Ethical Review Panel.

\section{Results}

Table 1 shows the medians for Goal Achievement and means for the other adherence variables across the three conditions, averaged across completed surveys from all participants who completed the study. Adherence across the different survey collection points was mostly consistent, with slightly more exercise carried out in the first and final surveys. 
Table 1. Comparison of outcomes by condition for the four adherence measurements

\begin{tabular}{|c|c|c|c|c|c|c|}
\hline & \multicolumn{2}{|l|}{$\begin{array}{l}\text { Music } \\
n=15\end{array}$} & \multicolumn{2}{|c|}{$\begin{array}{l}\text { Implementation } \\
\text { Intentions } \\
\qquad n=19\end{array}$} & \multicolumn{2}{|l|}{$\begin{array}{l}\text { Control } \\
n=16\end{array}$} \\
\hline & Median & & Median & & Median & \\
\hline Goal Achievement $^{\mathrm{a}}$ & 71.43 & & 78.57 & & 48.61 & \\
\hline & Mean & $S D$ & Mean & $S D$ & Mean & $S D$ \\
\hline Minutes & 282.02 & 111.97 & 284.10 & 207.31 & 220.31 & 121.33 \\
\hline Days & 4.76 & 1.26 & 4.13 & 1.70 & 3.57 & 1.19 \\
\hline Sessions ${ }^{b}$ & 5.65 & 1.84 & 5.35 & 2.97 & 3.94 & 1.44 \\
\hline $\begin{array}{l}\text { Number of sessions } \\
\text { planned per week }\end{array}$ & 8.07 & 2.72 & 5.82 & 2.47 & 7.59 & 2.67 \\
\hline
\end{tabular}

${ }^{a} p=.008$ (Mann-Whitney test) between implementation intentions and control groups and $p=.022$ (Mann-Whitney test) between music and control groups

${ }^{b} p=.030$ (Games-Howell) between music and control groups

\section{Goal Achievement}

A Kruskal-Wallis test showed a significant difference in Goal Achievement across the three conditions: (Group 1, $n=15$ : music, Group 2, $n=19$ : implementation intentions, Group 3, $n=16$ : control group), $\chi^{2}(2, n=50)=9.043, p=.011$. The implementation intentions group recorded higher scores than the music group, with the control group scoring lowest. Post-hoc Mann-Whitney U tests showed effects between the implementation intentions and control groups $(p=.008, r=.45$, medium effect size), and between the music and control groups $(p=.022, r=.41$, medium effect size $)$. We also found a positive correlation between Agreeableness and Goal Achievement ( $r=$ 
$.290, n=49, p=.044)$. There were no other correlations found between personality and adherence measures,

\section{Minutes}

The Minutes variable refers to the average duration of exercise in a survey week. The outcomes were slightly better for the implementation intentions group than the music group, with the control group achieving the shortest durations. The effect was small, however, and there was no statistically significant difference in the ANOVA: $F(2,47)=$ $.865, p=.428, \eta^{2}=.04$.

\section{Days}

The Days variable allowed comparison of the average number of days per week each group exercised during the survey weeks. Those with the music intervention exercised the most days per week, followed by the implementation intentions group, with the control group exercising on the fewest days. Differences were marginal: $F(2,47)=$ $2.691, p=.078, \eta^{2}=.10$.

\section{Sessions}

The Sessions variable refers to the average number of workouts over the survey weeks, including multiple sessions on a particular day. The music group carried out the most sessions, followed by the implementation intentions group, with the control group carrying out the fewest. There was a significant difference between groups. Levene's test was significant $(p=.034)$ so the Welch statistic was used: $F_{\text {Welch }}(2,30.22)=4.603$, 
$p=.018, \eta^{2}=.10$. The effect was between the music and control groups (GamesHowell: $p=.030)$.

\section{Choice of intervention}

Participants in the Music group listened primarily to Upbeat and Conventional music (6 participants), with 3 listening to Intense and Rebellious music and 1 to Rhythmic and Energetic music. One participant did not describe their music, while 4 participants described music too eclectic to categorise. Participants in the Implementation Intentions group provided 109 sentences across the surveys, with responses typically including one or two sentences. Nine participants used 'carrot' sentences and three used 'stick' sentences. Seven participants used sentences that could not be categorised as carrot or stick.

\section{Baseline measures and attrition}

Of the 99 participants recruited, 50 completed the study, 21 completed one or more exercise surveys but did not complete the study (part-completers), while 28 completed no exercise surveys (early withdrawers). These three groups were compared to investigate possible underlying factors: variables investigated were personality (supported by Rhodes et al., 2003), and age and BMI (supported by Bauman et al., 2012). The only significant difference was for Extraversion: $F(2,95)=5.124, p=.008$, $\eta^{2}=.10$ (medium effect). Completers and part-completers had the same mean score $(M$ $=3.90, S D=1.33$ and 1.60 respectively) and early withdrawers scored higher at $M=$ 4.93, $S D=1.60$. Significant differences were between early withdrawers and 
completers, and between early withdrawers and part-completers $(p=.009$ and $p=.040$ respectively: Tukey). There were no significant differences in age, BMI, personality or intended sessions per week between groups of participants assigned to each condition, therefore randomisation appears to have been successful.

A third of exercisers and a third of those not exercising regularly at the outset of the study were allocated to each condition. Attrition from the non-exercisers was higher than from the exercisers $(60.0 \%$ and $44.9 \%$ respectively), but there was no significant difference in attrition between exercisers and non-exercisers, nor between conditions. It also became apparent that some participants withdrew from the study but continued to exercise, therefore attrition cannot necessarily be assumed to indicate non-adherence.

\section{Zero Surveys}

Fifteen participants returned surveys with no exercise recorded (rather than not submitting a survey), indicating a lapse; ten went on to complete the study. Reasons given included tiredness, workload, illness, moving house and losing weight through dieting and thus not feeling a need to exercise. Six participants completed more than one zero survey: all six completed the study. Three completed one zero survey, followed by at least one survey included exercise before withdrawing, while two withdrew after the zero survey. The vast majority of zero surveys, indicating some kind of lapse, were followed by a return to exercise. 


\section{Discussion}

This study compared a pre-exercise music intervention with an implementation intentions intervention ('if... then...' sentences), and found a clear benefit for both interventions compared with a control group. The intervention groups outperformed the control group on all four measures of adherence, although not all differences were statistically significant. There were significant differences for Goal Achievement firstly between the implementation intentions and control groups, and secondly between the music and control groups. There were significant differences for Sessions between the music and control groups. Nevertheless, this demonstrates support for the first hypothesis. It should also be noted that the sample was predominantly female, while effects for adherence following playing music during exercise have been found in men or predominantly male samples.

It was anticipated that the music group would outperform the implementation intentions condition because of music working as a stimulus at multiple levels, and the music group exercised more frequently than the implementation intentions group. However, the differences between the two interventions were not significant on any measure of adherence, and the implementation intentions group exercised on average for 2 minutes longer each week than the music group.

The reason for the positive correlation between Agreeableness and Goal Achievement is not clear. There were no significant findings for other measurements of adherence and personality, suggesting that carrying out realistic plans, rather than setting overambitious goals or exercising to extremes, may underpin this. It may be that agreeable people are more likely than less agreeable people to take account of the demands of others and factor this into their plans. The correlation between Extraversion 
and increased likelihood of attrition was in the opposite direction to that anticipated based on Rhodes and Courneya (2003). They suggest that their participants found gyms stimulating which would appeal more to extraverts than introverts. It is possible that in the sample here, extraverts eschewed exercise environments (which were not limited to gyms, and may have been insufficiently stimulating) in favour of social activities not involving exercise.

The results indicated that most participants achieved substantially more than the UK recommended levels of cardiovascular exercise (NHS, 2011), which recommend 150 minutes of moderate or 75 minutes of vigorous exercise per week spread over several sessions, despite low levels of awareness of these targets found in the general population (Knox et al., 2013). The few not achieving this minimum set themselves lower targets, and achieved a smaller proportion of those targets. This may be due to perceptions of available time and reluctance to overcommit: however, setting higher goals may have a motivating effect, or help adherence through some other mechanism. This needs further investigation as it may help exercisers develop more effective plans if encouraged to set more ambitious targets. Plans do, nevertheless, vary considerably between studies. Of Rhodes et al.'s 290 participants (2003), 36\% set targets of fewer than 3 exercise sessions per week, while only 3 of the 50 participants in the current study (6\%) did likewise. It may be, therefore, that certain cohorts (Rhodes et al.'s participants were undergraduates) are more cautious in their ambitions, or that participants in the current study did not anticipate being measured against their targets. Non-exercisers' goals were for approximately one session fewer per week than regular exercisers, although the difference was not significant. Of the three participants setting targets of under three sessions per week, one was a regular exerciser prior to 
participation and the small numbers of participants setting low goals limit analysis of exerciser/non-exerciser differences.

While the amount of exercise was mostly consistent from one survey to the next, there were increased levels in the first and final surveys. This may be due to a 'novelty' effect motivating activity in the first survey. The increase for the final study was smaller, and might be attributable to concerns regarding not having exercised enough during the study, or through enhanced motivation due to 'sight of the finish line.' Whatever the reasons for the increased exercise at the beginning and end of the study, this effect is worthy of investigation because of its implications for short studies.

For the most part, a zero-exercise survey indicated a temporary lapse, with most zero surveys followed by a survey indicating a return to regular exercise. It is, however, possible that participants completing no exercise did not return a survey and withdrew at that point. The attrition was concentrated early in the study, with 28 of the 49 participants who withdrew completing no monthly surveys at all, although it is not clear how many continued to exercise. It is possible that the task of completing surveys was too onerous, and future studies might consider using SMS survey alerts and links as well as emails to maximise the ease of participation. The consequence is that it is difficult to draw conclusions about lapses from the data collected during the study.

Intervention use differed between the music and implementation groups, with the music group reporting using the intervention $80 \%$ of the time and the implementation intentions group reporting using the intervention $50 \%$ of the time. However, as implementation intentions may evoke strategy use which is not conscious, these differences may be smaller than the figures suggest, and these findings should be treated with caution. The difference was not found to be statistically significant. 
The study was limited by powering, and the attrition rate reduced the feasible amount of meaningful analysis - for example, to compare different groups looking at interactions with other variables. As the sample was self-selecting, participants were either already exercising or intending to do so, therefore intention and motivation was likely to be higher than in the population generally, limiting extrapolation. It would also have been useful to take more detailed measures of baseline exercise to achieve increased control in analyses, and also to collect data regarding duration of listening to music prior to exercising. Measurements of exercise intensity are currently difficult to carry out without affecting ecological validity, but developments in technology mean this will increasingly be possible, and this would add a further dimension to the findings. Further data regarding the activities of the music group, particularly location of listening, privacy (whether others were present), and equipment (for example speakers or headphones), could have provided further insight. This is recommended by Robb and Carpenter (2009), and Robb et al. (2010) in their method for classifying and reviewing music-based interventions, and could indicate the extent of intervention consistency and perhaps demonstrate a need for a more specific instruction to participants. Powering meant that it was not feasible to analyse by type of music listened to or sentence content. However, there were still important findings regarding the efficacy of music as a pre-exercise intervention.

Future research should explore the interventions with a larger sample, incorporating a group using both interventions, and investigating whether different people are more suited to different interventions, as well as the effects of different kinds of music and sentences. Measures of intensity would present more detail of activities, and as mobile apps improve and are more widely adopted, remote data collection will 
become easier. This allows 'real world' exercising, with an ad hoc element, rather than researchers measuring intensity in prescribed sessions or appointments.

Researchers extending this work should consider collecting data on intensity, using SMS technology, and combining music with other pre-exercise interventions. The rolling basis underpinning the study design could be extended for a longer period to achieve higher powering (the duration here was restricted by data collection deadlines).

\section{Conclusion}

This study suggests music has a beneficial effect on exercise adherence when used as a pre-exercise intervention. Exercisers who sometimes struggle with motivation may find music an effective, practical, easily applied and enjoyable strategy. Furthermore, results suggest that among regular exercisers, quantities of exercise carried out tend to exceed recommendations, and lapses are often temporary: along with the attrition patterns seen here, this suggests that non-adherence is most problematic early in an exercise programme, and interventions might most usefully be focussed at this point.

\section{Funding}

This research was funded by a Studentship from Keele University awarded to the first author. 


\section{References}

Ajzen I and Fishbein M (2005) The influence of attitudes on behaviour. In D Albarracin, B Johnson and M Zanna (Eds.), The Handbook of Attitudes (pp.173-221). Mahwah, New Jersey: Erlbaum.

Alter DA, O’Sullivan, M, Oh, PI, Redelmeier, DA, Marzolini, S, Liu, R, Forhan, M, Silver, M, Goodman JM and Bartel, LR (2015) Synchronized personalized music audio-playlists to improve adherence to physical activity among patients participating in a structured exercise program: a proof-ofprinciple feasibility study. Sports Medicine - Open 1(23). DOI: 10.1186/s40798-015-0017-9.

Bauman AE, Reis RS, Sallis JF, Wells JC, Loos RJF and Martin BW (2012) Correlates of physical activity: why are some people physically active and others not? The Lancet 380(9838): 258-271.

Baumeister R F, Vohs K D and Tice D M (2007) The strength model of self-control. Current Directions in Psychological Science 16(6): 351-355.

Bélanger-Gravel A, Godin G and Amireault S (2011) A meta-analytic review of the effect of implementation intentions on physical activity. Health Psychology Review 7(1): 23-54. DOI: 10.1080/17437199.2011.560095.

Bishop DT, Karageorghis CI and Loizou G (2007) A grounded theory of young tennis players' use of music to manipulate emotional state. Journal of Sport and Exercise Psychology 29: 584-607

Clark IN, Baker FA and Taylor NF (2015): The modulating effects of music listening on health-related exercise and physical activity in adults: a systematic review and narrative synthesis. Nordic Journal of Music Therapy. Published online. DOI: 10.1080/08098131.2015.1008558.

Cohen J and Cohen P (1983) Applied Multiple Regression/Correlation Analysis for the Behavioral Sciences. New Jersey/London: Lawrence Erlbaum Associates.

Conraads VM, Deaton C, Piotrowica E, Santaularia N, Teirney S, Piepoli MF ... Jaarsma T (2012) Adherence of heart failure patients to exercise: barriers and possible solutions. European Journal of Heart Failure 14: 451-458. DOI:10.1093/eurjhf/hfs048.

DeNora T (2000) Music in Everyday Life. Cambridge: Cambridge University Press.

Donaghy ME (2007) Exercise can seriously improve your mental health: Fact or fiction? Advances in Physiotherapy 9(2): 76-88. DOI: 10.1080/14038190701395838.

Edmunds J, Ntoumanis N and Duda JL (2007) Adherence and well-being in overweight and obese patients referred to an exercise on prescription scheme: A self-determination theory perspective. Psychology of Sport and Exercise 8: 722-740 DOI:10.1016/j.psychsport.2006.07.006.

Elliott D, Carr S, and Orme D (2005) The effect of motivational music on sub-maximal exercise. European Journal of Sport Science 5(2): 97-106. DOI: 10.1080/17461390500171310.

Garber CE, Blissmer B., Deschenes MR, Franklin BA, Lamonte MJ, Lee IM, Nieman DC, and Swain DP (2011) Quantity and quality of exercise for developing and maintaining cardiorespiratory, musculoskeletal, and neuromotor fitness in apparently healthy adults: guidance for prescribing 
exercise (American College of Sports Medicine position stand). Medicine \& Science in Sports \& Exercise 43(7): 1334-1359.

Gollwitzer PM (1999) Implementation Intentions: Strong effects of simple plans. The American Psychologist 54(7): 493-503.

Gollwitzer PM and Oettingen G (1998) The emergence and implementation of health goals. Psychology and Health 13(4): 687-715

Gollwitzer PM and Sheeran P (2006) Implementation intentions and goal achievement: a meta-analysis of effects and processes. Advances in Experimental Social Psychology 38: 69-119. DOI: 10.1016/S0065-2601(06)38002-1.

Gosling S, Rentfrow P and Swann w (2003) A very brief measure of the Big-Five personality domains. Journal of Research in Personality 37, 504-528.

Hallett RJ (2015) Music use and exercise: A mixed methods study of activity, autonomy and adherence (Doctoral Thesis). Keele University, Staffordshire, UK. Retrieved from http://eprints.keele.ac.uk/1182/

Hallett R and Lamont A (2015) How do gym members engage with music during exercise? Qualitative Research in Sport, Exercise and Health, 7(3), 411-427. DOI: 10.1080/2159676X2014.949835.

Hodges DA (2009). Bodily responses to music. In S Hallam, I Cross and M Thaut (Eds.), The Oxford Handbook of Music Psychology (pp. 121-130). Oxford: Oxford University Press.

Juslin P N, Harmat L and Eerola T (2013) What makes music emotionally significant? Exploring the underlying mechanisms. Psychology of Music 42(4): 599-623. DOI: 10.1177/0305735613484548

Juslin PN and Sloboda J (2010) Introduction: Aims, organization and terminology. In PN Juslin and J Sloboda (Eds.), Handbook of Music and Emotion: Theory, Research, Applications (pp. 3-12). Oxford: Oxford University Press.

Karageorghis C I and Priest D-L (2012) Music in the exercise domain: a review and synthesis (Part I). International Review of Sport and Exercise Psychology 5(1): 44-66. DOI: 10.1080/1750984X.2011.631026.

Karageorghis CI, Terry PC and Lane AM (1999) Development and initial validation of an instrument to assess the motivational qualities of music in exercise and sport: The Brunel Music Rating Inventory. Journal of Sports Sciences 17(9): 713-724. DOI: 10.1080/026404199365579.

Knox ECL, Esliger DW, Biddle SJH and Sherar LB (2013) Lack of knowledge of physical activity guidelines: can physical activity promotion campaigns do better? BMJ Open 2013(3): e003633. Retrieved from http://bmjopen.bmj.com/content/3/12/e003633.full.pdf+html.

Laukka P and Quick L (2013) Emotional and motivational uses of music in sports and exercise: A questionnaire study among athletes. Psychology of Music 41(2): 198-215. 
Mitchell L and MacDonald R (2012) Music and pain: Evidence from experimental perspectives. In

R.A.R. MacDonald, G. Kreutz and L. Mitchell (Eds.) Music, Health and Wellbeing. (pp. 230-238). Oxford: Oxford University Press.

Myers J (2008) The health benefits and economics of physical activity. Current Sports Medicine Reports 7(6): 314-316. DOI: 10.1249/JSR.0b013e31818ee179.

NHS (2011) Factsheet 4: physical activity guidelines for adults aged 19-64. Retrieved from http://www.nhs.uk/Livewell/fitness/Documents/adults-19-64-years.pdf

North AC, Hargreaves DJ and Hargreaves JJ (2004) Uses of music in everyday life. Music Perception, 22(1), 41-77.

Olson RL, Brush CJ, O'Sullivan DJ and Alderman BL (2015) Psychophysiological and ergogenic effects of music in swimming. Comparative Exercise Physiology 11(2): 79-87. DOI; 10.3920/CEP150003

Pallant J (2010) SPSS Survival Manual, $4^{\text {th }}$ Edition. Berkshire: McGraw Hill.

Rentfrow, P and Gosling, S (2003) The Do Re Mi's of Everyday Life: The Structure and Personality Correlates of Music Preferences. Journal of Personality and Social Psychology, 84(6), 1236-1256.

Rhodes RE, Courneya, KS and Jones, LW (2003) Translating Exercise Intentions into Behavior:

Personality and Social Cognitive Correlates. Journal of Health Psychology 8(4): 447-458: DOI: 10.1177/13591053030084004.

Rickard NS (2004) Intense emotional responses to music: a test of the physiological arousal hypothesis. Psychology of Music 32(4): 371-388.

Robb SL and Carpenter JS (2009) A Review of Music-based Intervention Reporting in Pediatrics. Journal of Health Psychology 14(4): 490-501. DOI: 10.1177/1359105309103568.

Robb SL, Carpenter JS and Burns DS (2010) Reporting Guidelines for Music-based Interventions. Journal of Health Psychology 16(2): 342-352. DOI: 10.1177/1359105310374781.

Saarikallio, S, Neiminen, S and Brattico, E (2013) Affective reactions to musical stimuli reflect emotional use of music in everyday life. Musicae Scientiae, 17(1), 27-39 DOI: 10.1177/1029864912462381

Salimpoor VN, Benovoy M, Longo G, Cooperstock JR and Zatorre RJ (2009) Rewarding aspects of music listening are related to degree of emotional arousal. PLOS One October 2009. DOI: 10.1371/journal.pone.0007487.

Shephard R (2003) Limits to the measurements of habitual physical activity by questionnaires. British Journal of Sports Medicine 37: 197-206.

Sloboda JA, O'Neill SA and Ivaldi A (2001) Functions of music in everyday life: an exploratory study using the Experience Sampling Method. Musicae Scientiae 5(1): 9-32.

Sniehotta F (2013) Keynote speech, British Psychological Society Division of Health Psychology Annual Conference (Brighton, UK). Accessed at http://www.youtube.com/watch?v=ySnSxIeu5uM $3 / 11 / 2015$ 
Sperandei S, Vierira MC and Reis, AC (2016) Adherence to physical activity in an unsupervised setting: Explanatory variables for high attrition rates among fitness centre members. Journal of Science and Medicine in Sport, in press, corrected proof, available online 28 Jan 2016. DOI:

10.1016/j/jsams.2015.12.522.

Stone D, Deci E and Ryan R (2009) Beyond talk: Creating autonomous motivation through selfdetermination theory. Journal of General Management, 34, 75-91.

Sport England (2013) Active People Survey 7. Retrieved from http://archive.sportengland.org/research/active_people_survey/active_people_survey_7.aspx.

Stanton R, Happell B, and Reaburn P (2014) The mental health benefits of regular physical activity, and its role in preventing future depressive illness. Nursing: Research and Reviews 2014(4): 45-53.

Stork MJ, Kwan MY, Gibala MJ and Martin Ginis KA (2014) Music enhances performance and perceived enjoyment of sprint interval exercise. Medicine and Science in Sports and Exercise 47(5): 1052-1060. DOI:10.1249/MSS. 0000000000000494.

Thoma M V, Ryf S, Mohiyeddin, C, Ehlert U and Nater UM (2012) Emotion regulation through listening to music in everyday situations. Cognition and Emotion 26(3): 550-560. DOI: $10.1080 / 02699931.2011 .595390$

Tice D M, Baumeister R F, Shmueli D, and Muraven M (2007) Restoring the self: Positive affect helps improve self-regulation following ego depletion. Journal of Experimental Social Psychology 43(3): 379-384. DOI: 10.1016/j.jesp.2006.05.007.

Tomarken AJ and Serlin RC (1986) Comparison of ANOVA alternatives under variance heterogeneity and specific noncentrality structures. Psychological Bulletin 99(1): 90-99.

Vrijens B, De Geest, S, Hughes DA, Przemyslaw K, Demonceau J, Ruppar T, ... Urquhart J (2012) A new taxonomy for describing and defining adherence to medications. British Journal of Clinical Pharmacology 73(5): 691-705. DOI: 10.1111/j.1365-2125.2012.04167.x

Warburton DE, Charlesworth S, Ivey A, Nettlefold L, and Bredin SSD (2010) A systematic review of the evidence for Canada's Physical Activity Guidelines for Adults. International Journal of Behavioural Nutrition and Physical Activity 7(39). Open Access: DOI:10.1186/1479-5868-7-39.

Zhu Z, Li J, Zhang B, Li Y and Zhang H (2015) The effect of motivation and positive affect on ego depletion: Replenishment versus release mechanism. International Journal of Psychology. Published online. DOI: 10.1002/ijop.12235. 